\section{Prediction of Early and Late Re-intubation: Is It a Crossed or Separate Line?}

\section{To the Editor:}

We read with interest the article by Miu et $\mathrm{al}^{1}$ on a very important issue regarding weaning from mechanical ventilation: the identification of predictors of re-intubation in subjects who have successfully completed a spontaneous breathing trial. We would like to comment on the selection of periods at risk for re-intubation used in this study. Two related aspects should be considered when selecting these periods for the prediction to be the most useful.

First, a prediction of risk for re-intubation assumes a near mechanistic relationship between the condition of the patient on the day of prediction and of extubation failure. How long does this relationship last? Does it include most patients at risk? Does it yield a sufficiently accurate prediction? Although more patients may require re-intubation within the first $24 \mathrm{~h}$ than in any subsequent day, the risk is likely to extend beyond this period. The authors indicate a median time to re-intubation of $22 \mathrm{~h}$, but the results suggest that only $41 \%$ of all re-intubated patients (155 of 379) were re-intubated in the first $24 \mathrm{~h}$. Time periods for risk of extubation failure of 48 and $72 \mathrm{~h}$ have been more commonly studied. ${ }^{2-5}$ In contrast, after a number of days, unforeseen conditions can develop in critically ill patients and be responsible for a new respiratory failure. ${ }^{3}$ Can an extubation failure 6 days after extubation be reasonably predicted? We suspect that, after a period of time postextubation, the relationship between the condition on extubation day and extubation failure weakens, the associated risk decreases, and a prediction of high risk for late reintubation becomes less accurate.

The second aspect to consider is the clinical implication of the prediction. A prediction of high risk for early re-intubation would allow the clinician either to delay extubation until the patient's condition further improves or to apply immediate, more intensive measures after extubation, as suggested by the authors, to minimize such risk. A prediction of high risk for late re-intubation would, however, have less clear implications. Options such as delaying extubation for several days, tracheostomy, and immediate extubation with prolonged intensive respiratory care could affect a sizable num- ber of patients, and resources. Knowing the accuracy of this latter prediction would be very important to assist with these decisions.

The period at risk for early re-intubation of $24 \mathrm{~h}$ selected in this study may not include a substantial proportion of re-intubations related to the patient condition on the prediction day. Analyzing the frequency of distribution of time to re-intubation could be informative to select a more inclusive early period at risk. Depending on its accuracy, a prediction for a longer early period could enhance its clinical implication.

The alternative period at risk selected, any time after extubation, may include re-intubations not directly related to the patient condition on prediction day and may be heavily influenced by a majority of re-intubations in the first few days, including the first $24 \mathrm{~h}$. Selecting mutually exclusive early and late periods at risk may better inform whether late re-intubations are predictable.

Juan B Figueroa-Casas MD Division of Pulmonary and Critical Care Medicine

Texas Tech University Health Sciences Center El Paso, Texas

Antonio M Esquinas MD PhD FAARC Intensive Care Unit Non-Invasive Ventilatory Unit Hospital Morales Meseguer Murcia, Spain

The authors have disclosed no conflicts of interest.

DOI: $10.4187 /$ respcare. 03348

\section{REFERENCES}

1. Miu T, Joffe AM, Yanez ND, Khandelwal N, Dagal AH, Deem S, Treggiari MM. Predictors of re-intubation in critically ill patients. Respir Care 2014;59(2):178-185.

2. Thille AW, Harrois A, Schortgen F, BrunBuisson C, Brochard L. Outcomes of extubation failure in medical intensive care unit patients. Crit Care Med 2011;39(12): 2612-2618.

3. Epstein SK, Ciubotaru RL, Wong JB. Effect of failed extubation on the outcome of mechanical ventilation. Chest 1997;112(1): 186-192.

4. Khamiees M, Raju P, DeGirolamo A, Amoateng-Adjepong Y, Manthous CA. Predictors of extubation outcome in patients who have successfully completed a spontaneous breathing trial. Chest 2001;120(4):12621270.
5. Liu Y, Wei LQ, Li GQ, Lv FY, Wang H, Zhang YH, Cao WL. A decision-tree model for predicting extubation outcome in elderly patients after a successful spontaneous breathing trial. Anesth Analg 2010;111(5): 1211-1218.

\section{Prediction of Early and Late Re-intubation: Is It a Crossed or Separate Line?-Reply}

\section{In Reply:}

We thank Drs Figueroa-Casas and Esquinas for their important comments regarding the prediction of early and late re-intubation.

We agree that there are multiple potential mechanisms and pathways leading to extubation failure. Our data suggest that there are several patient characteristics that predict the likelihood of requiring re-intubation. We also concur that these patient characteristics do not necessarily resolve and represent an ongoing risk of extubation failure.

In terms of defining early and late events, we chose $24 \mathrm{~h}$ because more than half of the patients would have failed extubation within this window. In addition, this time frame might have implications regarding the decision to transfer out of the ICU. If high-risk patients are immediately discharged postextubation, they may require rapid readmission to the ICU. It would make clinical sense to extend the ICU monitoring $\sim 24 \mathrm{~h}$ after extubation; however, an increased risk of extubation failure beyond $24 \mathrm{~h}$ would be unlikely to justify a longer ICU stay. For patients with long-term failure, the ability to identify those at risk may not only allow targeting specific therapies but also potentially allow informed discussions on prognosis involving the decision to (re)intubate or perform a tracheostomy.

Prediction of extubation failure can be thought of as the prediction of mortality based on physiologic variables and chronic health evaluation noted on ICU admission, which are the basis of the Acute Physiology and Chronic Health Evaluation (APACHE) score calculation. When predicting mortality on ICU admission, it is not possible to account for all the events or subsequent complications that might occur during the ICU stay. Nonetheless, certain predisposing factors can be identified, and there is value in identifying high-risk patients, particularly if certain risk factors are potentially modifiable. For high-risk patients, for example, a 